\title{
DISEÑO DE UNA PINZA SUBACTUADA HÍBRIDA SOFT-RIGID CON SENSORES HÁPTICOS PARA INTERACCIÓN FÍSICA ROBOT-HUMANO
}

\author{
Trinidad Sánchez-Montoya, Juan M. Gandarias, Francisco Pastor, \\ Antonio J. Muñoz-Ramírez, Alfonso J. García-Cerezo, Jesús M. Gómez-de-Gabriel \\ Departamento de Ingeniería de Sistemas y Automática, Universidad de Málaga \\ \{trinisanmon,jmgandarias,fpastor,aj,ajgarcia,jesus.gomez\}@uma.es
}

\begin{abstract}
Resumen
Las manos robóticas sub-actuadas poseen una gran adaptabilidad que les permite el agarre de objetos de diferentes formas y tamaños. Por otro lado, el desarrollo de robots blandos (soft robotics) para la interacción física segura con personas constituye un importante campo de desarrollo de la robótica de los últimos años. Sin embargo, es difícil encontrar trabajos que integren ambos conceptos. En este artículo se presenta el desarrollo de una pinza con dedos rígidos y rígido-flexible de dos grados de libertad, sub-actuados. En este último, además, se incorpora un sensor táctil de alta resolución que permite al robot disponer de la información relativa de las distribuciones de presión durante el agarre. Además, se presentan y comparan los resultados del comportamiento de dos dedos híbridos con superficies flexibles de distintos materiales en términos de flexibilidad y distribución de presiones cuando se agarra el brazo de una persona por la muñeca.
\end{abstract}

Palabras clave: interacción física robot-humano, pinza sub-actuada, soft robotics.

\section{Introducción}

En los últimos años ha aumentado el interés por la introducción de robots en tareas que implican la interacción con humanos, desde la contribución en actividades industriales [1] hasta el cuidado o asistencia de personas [2] o la creación de prótesis robotizadas [3]. La presencia de robots en numerosos ámbitos ha tenido repercusiones beneficiosas para la sociedad, no solamente facilitando tareas físicas sino también sociales [4].

Un campo destacable de estudio es la colaboración de robots en tareas de búsqueda y rescate de personas [5], donde la intervención de robots puede suponer una mejora en la eficiencia y seguridad de la tarea. Para llevar a cabo cualquier interacción física humano-robot (pHRI) es necesario, en primer lugar, garantizar la seguridad de las personas [6]. El desarrollo de sistemas de agarre adaptativos y de rigidez variable, tales

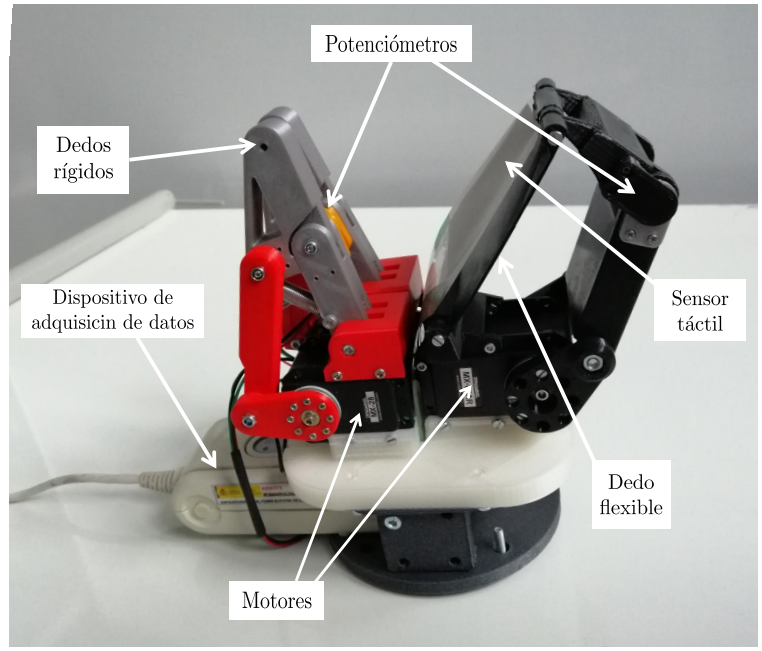

Figura 1: Prototipo final de la pinza subactuada hibrida de tres dedos. La pinza incluye sensores hápticos propioceptivos que permiten conocer la posicion de las articulaciones subactuadas de los dedos y un sensor táctil en el dedo flexible para obtener la distribución de la presión ejercida en el agarre.

como los presentados en $[7,8]$ resultan esenciales para pHRI. En $[9,10]$ se presenta la idea de que un robot sea capaz de ayudar a víctimas de catástrofes naturales realizando un reconocimiento de su antebrazo y tomando mediciones de sus constantes vitales mediante la colocación de sensores biométricos.

Para llevar a cabo este tipo de tareas es necesario disponer de una mano o pinza robótica con un diseño adaptativo que presente a la vez un agarre firme de la persona y pueda controlar su fuerza y movimientos para garantizar la seguridad. En $[11,12,13]$ se utiliza información de la presión ejercida durante el agarre que, mediante técnicas de aprendizaje automático (Machine Learning), se puede utilizar para obtener información de alto nivel, clasificar el contacto, e incluso reconocer objetos.

Para el desarrollo de pinzas robóticas adaptativas se han obtenido buenos resultados mediante la implementación de sistemas subactuados $[14,15]$ y 
el uso de materiales flexibles [16, 17], buscando un sistema de prototipado y fabricación asequible mediante su producción a través de impresión 3D [18]. También ha sido contemplado un diseño modular en el que la pinza queda conformada por dedos individuales que trabajan de manera coordinada $[19,20]$. Por otro lado, hasta la fecha pueden encontrarse algunos ejemplos de sistemas robóticos que utilizan sensores de presión para garantizar la seguridad en pHRI, como en el caso de el modelo diseñado para la asistencia para vestir a personas en [21]. Sin embargo no ha sido llevado a cabo aún un diseño y desarrollo de un robot capaz de reconocer a una persona e interaccionar físicamente de manera directa con ella.

En este artículo se presenta una solución novedosa para tareas de pHRI en las que el robot debe agarrar a una persona. Para ello se propone un diseño de pinza robótica híbrida subactuada que integra materiales rígidos y flexibles para garantizar la adaptabilidad y ergonomía del sistema a la vez que se mantiene una estructura suficientemente resistente. La pinza dispone de dos dedos rígidos subactuados y un dedo híbrido en el que la superficie de contacto con el objeto está fabricada con materiales flexibles. Todas las articulaciones pasivas cuentan con sensores propioceptivos que permiten conocer el ángulo para obtener información de la forma y tamaño del objeto agarrado. Además, se integra un array de sensores táctiles en la superficie flexible que permite obtener la distribución de presión durante el agarre. El prototipo final se muestra en la Figura 1.

La estructura de este artículo es la siguiente: En la sección 2 se presenta el modelado del sistema mecánico de la pinza, su diseño mediante software de modelado 3D y su fabricación y montaje a través de impresión 3D. Tras esto, en la sección 3, se trata la caracterización y validación de la pinza creada. Finalmente, en la sección 4, se exponen las conclusiones obtenidas.

\section{Diseño}

\subsection{Modelo cinemático}

Para el diseño de la pinza se busca que ésta sea capaz de agarrar un objeto adaptándose a su geometría para poder mapear de este modo la presión ejercida sobre cada punto de la superficie del objeto. En el caso de una pinza con dedos rígidos el contacto con el objeto de agarre tiene un carácter más puntual y la fuerza ejercida por la pinza se reparte de manera no homogénea. Para evitar esto se busca que la pinza cuente con un dedo con una superficie flexible que pueda adaptar su curvatura libremente al objeto agarrado. Los

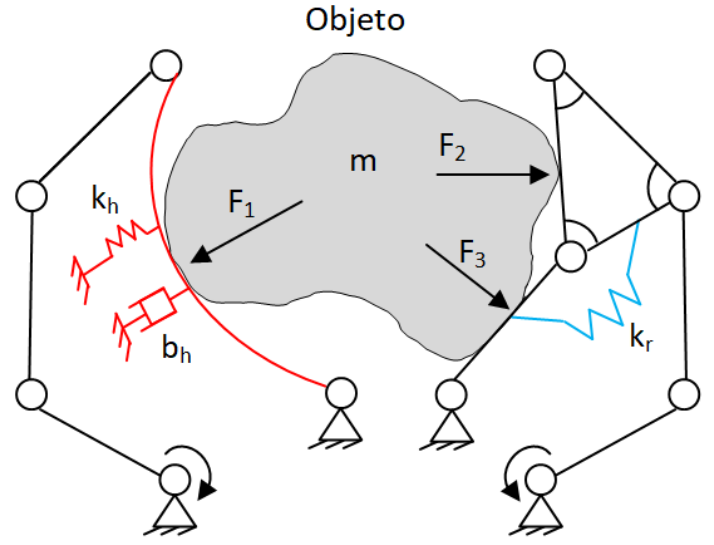

Figura 2: Modelo simplificado de la pinza híbrida. Dedo híbrido rígido-flexible a la izquierda, con componente elástica y amortiguadora que introduce la pieza flexible (en rojo) al ejercerse sobre ella una fuerza $\mathrm{F}$, y dedo completamente rígido a la derecha, al que se le incorpora un muelle externo (en azul).

extremos de la pieza flexible se unen a la pinza mediante articulaciones que permiten el giro de ésta respecto al resto de eslabones.

El modelo simplificado de la pinza se muestra en la Figura 2, y consta de dos dedos subactuados rígidos, y uno híbrido rígido-flexible que incorpora una pieza flexible como superficie de contacto con el objeto de agarre. Esta disposición busca garantizar un agarre firme proporcionado por la combinación de la acción de los dos dedos con superficies rígidas, junto con la acción del dedo híbrido, cuya superficie flexible se deforma en función de la geometría del objeto agarrado y de la fuerza ejercida. Todos los dedos de la pinza son subactuados, simplificando con ello el sistema de actuación.

En el caso de los dedos rígidos se introduce un elemento elástico (muelle) que obliga al dedo a permanecer completamente estirado cuando no se está aplicando una fuerza interna, es decir, cuando no se está agarrando ningún objeto. Por otro lado, en el caso del dedo híbrido, será la componente elástica del propio material flexible la que devolverá el dedo a la posición de referencia. El dedo híbrido está formado por tres eslabones rígidos y uno flexible, mientras que cada dedo rígido lo conformarán cuatro eslabones rígidos. A estos eslabones hay que sumar el eslabón fijo de la base de la pinza.

\subsection{Modelado 3D}

El diseño por el que finalmente se opta tras el estudio de posibles variaciones es el mostrado en la 


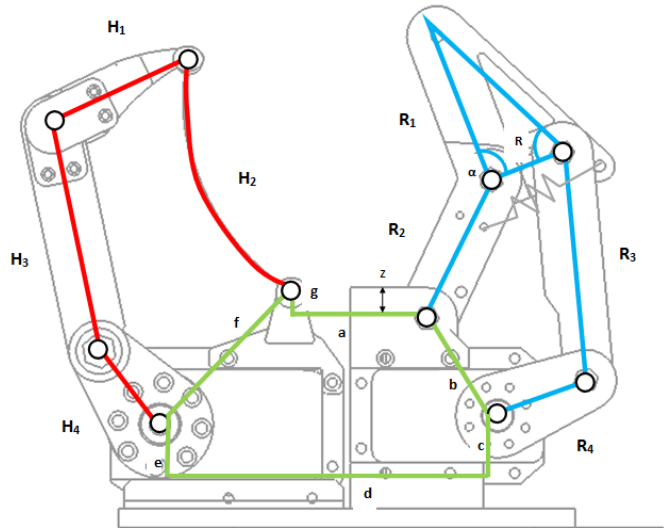

Figura 3: Modelo de la pinza híbrida en el que se nombran sus componentes para ser definidas sus dimensiones. Dedo híbrido rígido-flexible a la izquierda, en rojo, y dedo completamente rígido a la derecha, en azul. Base de la pinza en verde.

Figura 3.En este diseño los eslabones se dimensionan de forma que el sistema sea resistente y pueda adaptarse a la pieza flexible. Se tiene también en consideración la aplicación que se le quiere dar a la pinza (potencialmente el objeto de agarre será un antebrazo humano), por lo que las dimensiones deben permitir el agarre de un objeto de estas dimensiones y características. Se busca igualmente que haya suficiente distancia entre la pieza flexible y los eslabones rígidos para que estos no impidan que la superficie flexible se curve o adapte.

El espesor de la pieza flexible se elige de tal forma que la elasticidad y flexibilidad obtenidas permitan a la pinza moverse correctamente. Además de dimensionar su espesor se contemplan las dimensiones del sensor de presión que se utilizará de forma que la pieza flexible lo abarque en su totalidad. Para las uniones de los eslabones rígidos con el flexible se quiere conseguir una articulación que interfiera lo menos posible en la lectura de presiones, por lo que estas articulaciones se sitúan fuera de los límites del sensor de presión.

Una vez recogidas las características de diseño de la pinza se realiza su modelado 3D mediante el software SolidWorks 2018 según las dimensiones recogidas en la tabla 1 , y de acuerdo a la nomenclatura definida en la Figura 3.

Con estas dimensiones se realiza el modelo definitivo en 3D, mostrado en la Figura 4. Pueden observarse en rojo las piezas que cubren al potenciómetro que medirá el ángulo entre los eslabones $H_{1}$ y $H_{3}$. En la parte inferior de la base se encuentra el sistema de adquisición de datos del sensor de presión. Los dos dedos rígidos permiten que el agarre pueda adaptarse mejor a la geometría del objeto cogido y no se pierda infor-

\begin{tabular}{cc|cc}
\hline Parámetro & Valor & Parámetro & Valor \\
\hline \hline$a$ & $35 \mathrm{~mm}$ & $R$ & $20 \mathrm{~mm}$ \\
\hline$b$ & $30 \mathrm{~mm}$ & $R_{1}$ & $45 \mathrm{~mm}$ \\
\hline$c$ & $25 \mathrm{~mm}$ & $R_{2}$ & $40 \mathrm{~mm}$ \\
\hline$d$ & $90 \mathrm{~mm}$ & $R_{3}$ & $60 \mathrm{~mm}$ \\
\hline$e$ & $25 \mathrm{~mm}$ & $R_{4}$ & $27 \mathrm{~mm}$ \\
\hline$f$ & $55 \mathrm{~mm}$ & $H_{1}$ & $40 \mathrm{~mm}$ \\
\hline$g$ & $8 \mathrm{~mm}$ & $H_{2}$ & $70 \mathrm{~mm}$ \\
\hline$z$ & $8 \mathrm{~mm}$ & $H_{3}$ & $60 \mathrm{~mm}$ \\
\hline$\alpha$ & $90^{\circ}$ & $H_{4}$ & $27 \mathrm{~mm}$ \\
\hline
\end{tabular}

Tabla 1: Dimensiones que delimitan el diseño de pinza realizado.

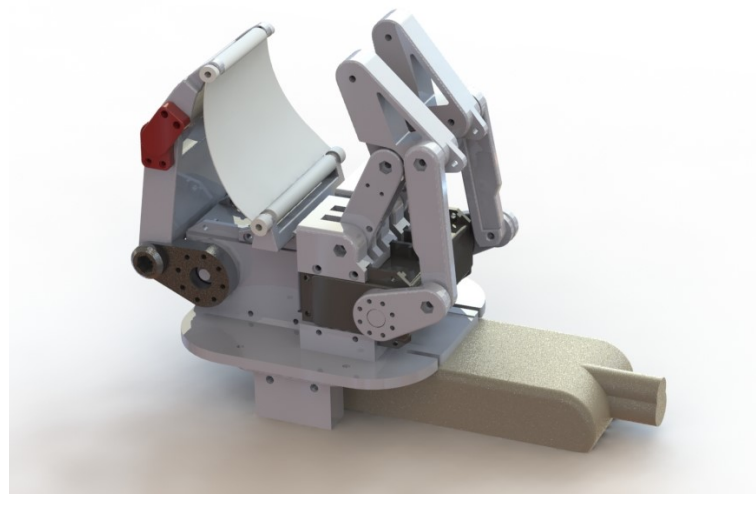

Figura 4: Modelado 3D en SolidWorks de la pinza con el dedo híbrido a la izquierda y dos dedos rígidos a la derecha. En la base de la pinza se encuentra sistema de adquisición de datos del sensor de presión.

mación del sensor de presión.

\subsection{Implementación}

El montaje del sistema completo se muestra en la Figura 1. El actuador para el dedo híbrido es un servomotor Dynamixel $M X-64 A R$, mientras que los actuadores de los dedo rígidos son servomotores Dynamixel $M X-28$, ambos de Robotis.

La impresión 3D de las piezas rígidas se realiza en PLA mediante una impresora $B C N 3 D$ sigma. La configuración que se escoge para la impresión es: $0,2 \mathrm{~mm}$ de altura de capa, $1,2 \mathrm{~mm}$ de grosor de las capas externas verticales (Shell thickness), $1 \mathrm{~mm}$ para las capas externas horizontales y una densidad del $20 \%$. Se imprime a una velocidad de $60 \mathrm{~mm} / \mathrm{s}$, a una temperatura de $205^{\circ}$ para el extrusor y de $55^{\circ}$ para la cama.

Por otro lado, se han diseñado y fabricado dos piezas flexibles (ver Figura 5) con distintos materiales, así la pieza de la izquierda se ha fabricado con PETG con un módulo de ruptura de $69 \mathrm{MPa}$ y resistencia a la tracción de $50 \mathrm{MPa}$, 


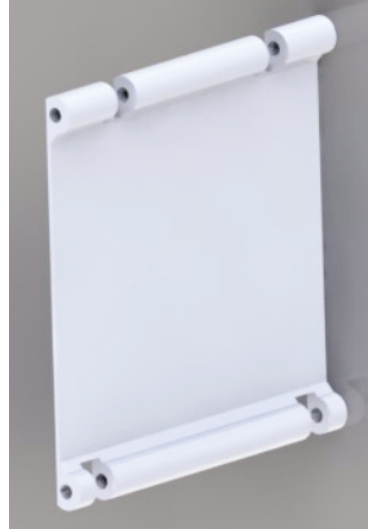

(a)

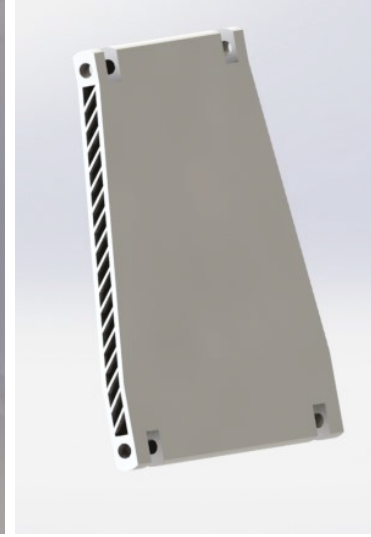

(b)
Figura 5: Diseño de la pieza flexible con PETG (a). Como este material tiene cierta flexibilidad pero no es muy elevada se diseña con una superficie de $1 \mathrm{~mm}$ de espesor. Diseño de pieza flexible con material FLEX (b). Al tener más flexibilidad que el PETG, es necesario que el diseño incluya una estructura interna que permita conseguir cierta rigidez, con un total de $5 \mathrm{~mm}$ de espesor.

un copolímero procedente de la combinación del Tereflatato de Ploietireno (PET) con glicol mediante un proceso de glicolizado; mientras que la pieza de la derecha se ha fabricado con FLEX, un elastómero termoplástico de 93A dureza (shore A) y $40 \mathrm{MPa}$ de resistencia a la tracción.

Sobre la superficie de contacto de estas piezas se ha incorporado un sensor táctil flexible de alta resolución, en concreto el modelo 5051 de Tekscan. Este sensor posee 1936 sensores resistivos distribuidos en una matriz de $44 \mathrm{x} 44$ tactels con una densidad de 62 tactels $/ \mathrm{cm}^{2}$.

\section{Caracterización de la pinza}

Para poder obtener un modelo que represente el comportamiento de la pinza se lleva a cabo una caracterización de las piezas flexibles impresas mediante la aplicación de un esfuerzo a flexión de estas y mediante la toma de imágenes táctiles a partir de las lecturas de presión del sensor mientras la pinza agarra un antebrazo.

\subsection{Relación fuerza-desplazamiento}

Una de las formas de caracterizar el comportamiento de la superficie flexible del dedo híbrido consiste en obtener la relación fuerzadesplazamiento. Para medir esta relación se ha realizado un experimento en el que se ejerce una fuerza en medio del dedo flexible en dirección perpendicular a la superficie, y se mide la deformación

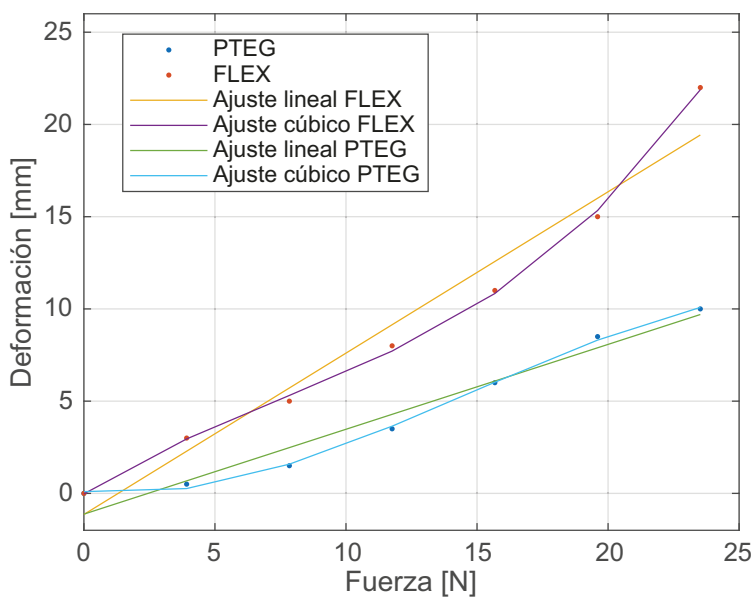

Figura 6: Representación de las curvas de comportamiento a flexión de la piezas flexibles de material FLEX y de PETG.

producida. Este experimento es similar al dibujo de la Figura 2, y permite visualizar la diferencia del comportamiento a flexión de las dos piezas flexibles.

En la Figura 6 se muestran las deformaciones de la pieza impresa en material FLEX y de la pieza impresa en PTEG frente a la acción de fuerzas perpendiculares a su superficie de agarre. En la imagen se representan, además de los datos obtenidos en los experimentos, un ajuste lineal y un ajuste de tercer orden de las curvas de comportamiento de las piezas.

$$
\begin{gathered}
\delta_{F L E X}^{l}(F)=0.87 F-1.1 \\
\delta_{P E T G}^{l}(F)=0.46 F-1.1 \\
\delta_{F L E X}^{p}(F)=0.0018 F^{3}-0.042 F^{2}+0.89 F-0,024 \\
\delta_{P E T G}^{p}(F)=0.0012 F^{3}-0.05,1 F^{2}-0.14 F-0,095
\end{gathered}
$$

Donde $F$ representa a la fuerza ejercida perpendicular a la superficie de las piezas, y $\delta_{P E T G}^{i} \mathrm{y}$ $\delta_{F L E X}^{i}$ representan la deformación de las piezas de PETG y FLEX, respectivamente. El superíndice $l$ hace referencia al resultado de la regresión lineal, mientras que el superíndice $p$ hace referencia al resultado de la regresión polinomial de tercer orden.

\subsection{Distribución de presiones}

Para obtener información sobre la adaptabilidad de la superficie flexible cuando se está agarrando un antebrazo, se ha realizado un experimento en el que se han tomado diferentes imágenes de presión realizando agarres del brazo de un sujeto con las piezas de PETG y FLEX. La Figura 7 muestra una fotografía realizada durante el experimento. 


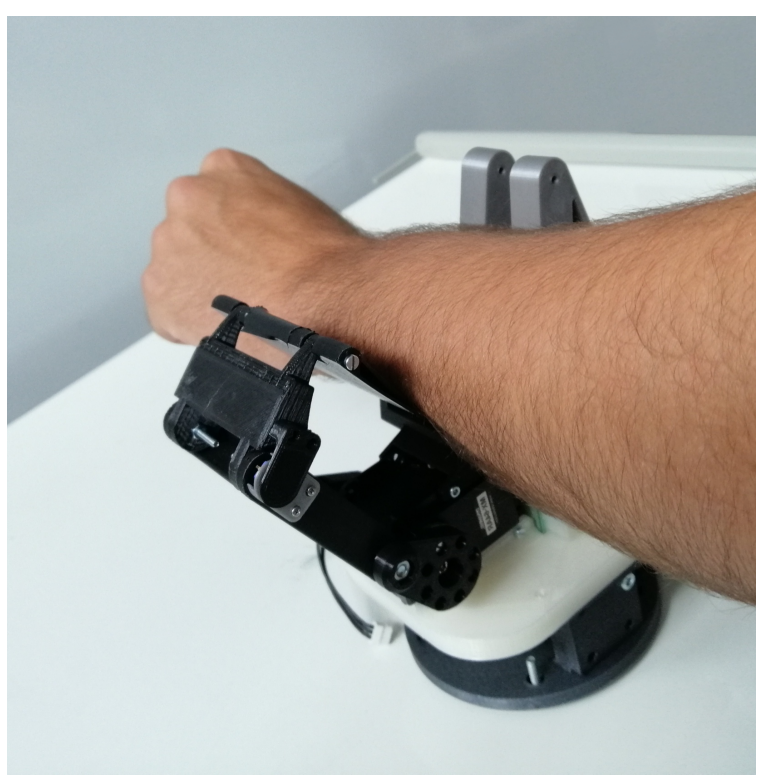

Figura 7: Fotografía de la pinza con un brazo posicionado en el lugar de agarre.

En la Figura 8 se muestran las matrices de lecturas de presión del sensor sobre las piezas flexibles para un caso en el que la pinza no ejerce y ejerce su cierre al rededor del brazo.

\subsection{Discusión de los resultados}

En base a las curvas fuerza/desplazamiento obtenidas, se puede asegurar que la pieza de material FLEX posee mayor flexibilidad que la pieza de material PETG. Además, al realizar el experimento, se ha comprobado que la pieza de material FLEX es capaz de soportar fuerzas superiores a $25 \mathrm{~N}$, sin embargo, a partir de ese valor, la pieza de PETG se puede llegar a romper.

Por otro lado, a partir de los resultados obtenidos de las imágenes de presión se puede obtener la superficie de contacto dividiendo el número de tactels distintos de 0 entre la densidad del sensor $\kappa$, en este caso $\kappa=62$ tactels $/ \mathrm{cm}^{2}$. Tal y como se muestra en la siguiente ecuación, en la que $\mathcal{I}(t)$ es el valor del tactel $t$ de la imagen de presión $\mathcal{I}$, y $\tau$ es el número total de tactels del sensor, en este caso, $\tau=1936$ tactels.

$$
S=\frac{\sum_{t=0}^{\tau} \mathcal{I}(t) \neq 0}{\kappa}
$$

En el caso de la pieza de material FLEX se ha medido un área de contacto de $66,12 \mathrm{~cm}^{2}$ cuando la pinza se cierra sobre el brazo. Sin embargo, para el caso de la pieza de PTEG se obtiene un área de contacto de $40,94 \mathrm{~cm}^{2}$, por lo que se demuestra que la pieza de material FLEX se adapta mejor al objeto en contacto. Sin embargo, en las imágenes táctiles de la Figura 8 puede observarse que, debido a esta mayor adaptabilidad, es más difícil distinguir la forma del antebrazo agarrado, ya que gran parte de la superficie del sensor está en contacto con el brazo, a diferencia de lo que ocurre con el PETG.

\section{Conclusiones}

En este trabajo se ha presentado un modelo de pinza robotizada híbrida rígida-flexible, subactuada y sensorizada para tareas de pHRI que requieran manipular extremidades humanas, centrándonos en un diseño apto para el agarre del antebrazo. Este modelo combina las características de agarre que ofrecen tanto las pinzas rígidas subactuadas como las pinzas blandas. Se han especificado las condiciones de diseño y se ha presentado el modelo 3D de la pinza. Además, se ha presentado el montaje definitivo, con dos dedos rígidos subactuados y un dedo híbrido con la superficie de contacto flexible. Se han realizado experimentos de caracterización y validación del comportamiento de la pinza con dos piezas flexibles de diferentes materiales y estructuras. Los resultados demuestran que tanto el PETG como el FLEX son aptos para el agarre y manipulación del antebrazo de personas, sin embargo, se ha demostrado que con el FLEX se obtiene mayor flexibilidad, robustez y superficie de contacto. Sin embargo, la mayor flexibilidad del FLEX puede ser un impedimento para el agarre del antebrazo, ya que ambas superficies son deformables. En futuros trabajo se realizarán experimentos con más materiales y diseños que proporcionen distinta rigidez a fin de encontrar un diseño óptimo para la manipulación de extremidades humanas. Por otro lado, se investigará la posibilidad de integrar dedos con dureza y fricción variables.

\section{Agradecimientos}

Este trabajo ha sido financiado por los proyectos nacionales DPI2015-65186-R, RTI2018-093421-BI00, la Universidad de Málaga y por la ayuda BES2016-078237 del Fondo Social Europeo FSE.

\section{English summary}

\section{DESIGN OF A HYBRID SOFT- RIGID UNDERACTUATED GRIP- PER WITH HAPTIC SENSORS FOR PHYSICAL HUMAN-ROBOT INTERACTION}

\author{
Abstract \\ Underactuated robotic hands or grippers is \\ one of the greatest achievements in robotic
}



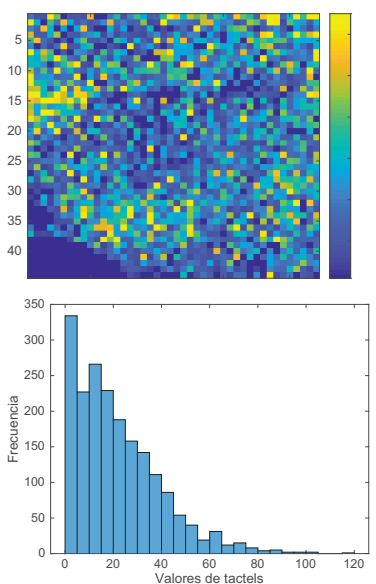

(a)
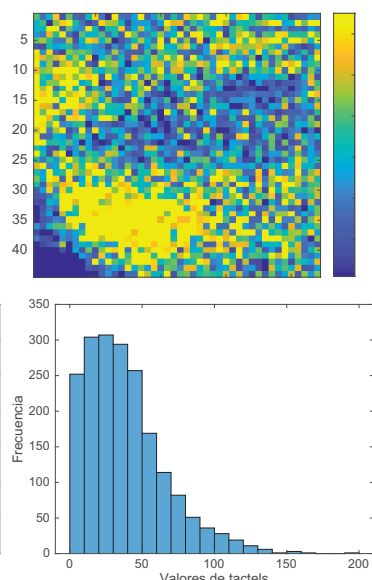

(b)
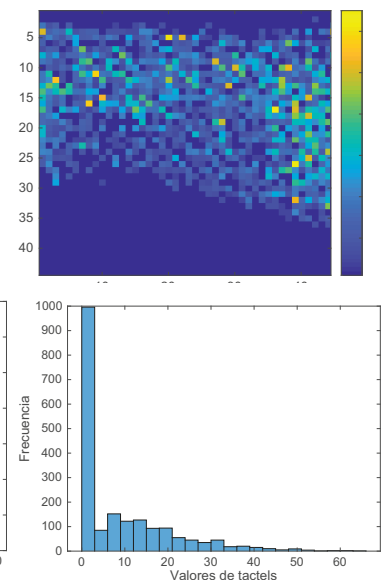

(c)
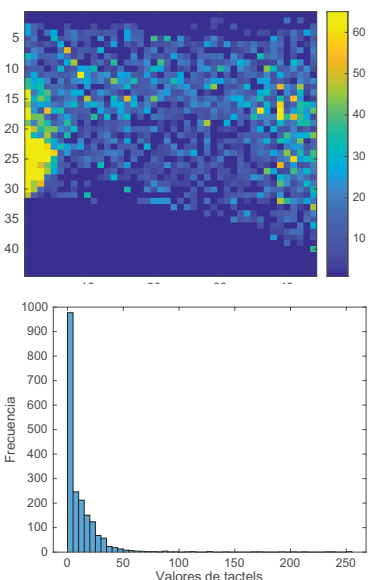

(d)

Figura 8: Lectura e histograma del sensor de presión para la pieza en material FLEX sin que la pinza se cierre, posicionando sobre ella un antebrazo (a). Lectura e histograma del sensor de presión para la pieza en material FLEX con la pinza cerrándose sobre un antebrazo (b). Lectura e histograma del sensor de presión para la pieza en material PETG sin que la pinza se cierre, posicionando sobre ella un antebrazo (c). Lectura e histograma del sensor de presión para la pieza en material PETG con la pinza cerrándose sobre un antebrazo (d).

manipulation of the last years due to the adaptability to grasp objects with different shape, size and stiffness. On the other hand, the development of soft robots for intrinsically safe physical Human-Robot Interaction (pHRI) has also become one of the most important topics in robotics in the last decade. However, it is hard to find works that integrates both concept in one gripper. In this work, a new gripper with two underactuated fingers and one flexible is presented. Furtheremore, the flexible finger integrates a high-resolution tactile sensor, so that the robot have the information of pressure distributions while grasping an object. Furtheremore, two different flexible fingers made of different materials are compared in terms of flexibility and pressure distribution when grasping a human forearm.

Keywords: physical Human-Robot Interaction (pHRI), underactuated gripper, soft robotics.

\section{Referencias}

[1] K. Harada, K. Nagata, J. Rojas, I. G. Ramirez-Alpizar, W. Wan, H. Onda, and
T. Tsuji, "Proposal of a shape adaptive gripper for robotic assembly tasks," Advanced Robotics, vol. 30, no. 17-18, pp. 1186-1198, 2016.

[2] D. Gallenberger, T. Bhattacharjee, Y. Kim, and S. S. Srinivasa, "Transfer depends on acquisition: Analyzing manipulation strategies for robotic feeding," in ACM/IEEE International Conference on Human-Robot Interaction (HRI), pp. 267-276, 2019.

[3] B. Chen, Q. Wang, and L. Wang, "Adaptive slope walking with a robotic transtibial prosthesis based on volitional emg control," IEEE/ASME Transactions on Mechatronics, vol. 20, no. 5, pp. 2146-2157, 2015.

[4] J. Fasola and M. J. Mataric, "Using socially assistive human-robot interaction to motivate physical exercise for older adults," Proceedings of the IEEE, vol. 100, no. 8, pp. 2512-2526, 2012.

[5] R. González, F. Rodríguez, and J. L. Guzmán, "Robots móviles con orugas historia, modelado, localización y control," Revista Iberoamericana de Automática e Informática Industrial RIAI, vol. 12, no. 1, pp. $3-12,2015$.

[6] S. A. Bowyer and F. Rodriguez y Baena, "Dissipative control for physical human-robot interaction," IEEE Transactions on Robotics, vol. 31, no. 6, pp. 1281-1293, 2015 . 
[7] A. H. Memar, N. Mastronarde, and E. T. Esfahani, "Design of a novel variable stiffness gripper using permanent magnets," in IEEE International Conference on Robotics and Automation (ICRA), pp. 2818-2823, 2017.

[8] A. Stilli, L. Grattarola, H. Feldmann, H. A. Wurdemann, and K. Althoefer, "Variable stiffness link (vsl): Toward inherently safe robotic manipulators," in IEEE International Conference on Robotics and Automation (ICRA), pp. 4971-4976, 2017.

[9] F. J. Ruíz-Ruíz, J. M. Gandarias, A. J. Muñoz-Ramírez, A. J. GarcíaCerezo, F. Pastor, and J. M. Gomez-de Gabriel, "Monitorización de víctimas con manipuladores aéreos en operaciones de búsqueda y rescate," in XXXIX Jornadas de Automática, Badajoz, 092018.

[10] J. M. Gómez-de-Gabriel, J. M. Gandarias, F. J. Pérez-Maldonado, F. J. García-Núñcz, E. J. Fernández-García, and A. J. GarcíaCerezo, "Methods for autonomous wristband placement with a search-and-rescue aerial manipulator," in IEEE/RSJ International Conference on Intelligent Robots and Systems (IROS), pp. 7838-7844, 2018.

[11] S. Funabashi, S. Morikuni, A. Geier, A. Schmitz, S. Ogasa, T. P. Torno, S. Somlor, and S. Sugano, "Object recognition through active sensing using a multi-fingered robot hand with 3d tactile sensors," in 2018 IEEE/RSJ International Conference on Intelligent Robots and Systems (IROS), pp. 2589-2595, Oct 2018.

[12] J. Gandarias, J. Gomez-de Gabriel, and A. J. García-Cerezo, "Enhancing perception with tactile object recognition in adaptive grippers for human-robot interaction," Sensors, vol. 18, p. 692, 022018.

[13] J. M. Gandarias, A. J. García-Cerezo, and J. M. Gómez-de-Gabriel, "CNN-based methods for object recognition with highresolution tactile sensors," IEEE Sensors Journal, 2019.

[14] A. Velázquez-Sánchez, E. Lugo-González, C. Torres-San-Miguel, and E. Merchán-Cruz, "Síntesis de un mecanismo subactuado a partir de la función decriptiva del dedo índice," Científica, vol. 13, pp. 95-103, 2009.

[15] B. Gao, S. Yang, H. Jin, Y. Hu, X. Yang, and J. Zhang, "Design and analysis of underactuated robotic gripper with adaptive fingers for objects grasping tasks," in IEEE International Conference on Robotics and Biomimetics (ROBIO), pp. 987-992, 2016.

[16] C. Basson, G. Bright, and A. Walker, "Testing flexible grippers for geometric and surface grasping conformity in reconfigurable assembly systems," South African Journal of Industrial Engineering, vol. 29, pp. 128 - 142, 002018.

[17] D. Petković, S. Shamshirband, N. B. Anuar, A. Q. M. Sabri, Z. B. Abdul Rahman, and N. D. Pavlović, "Input displacement neuro-fuzzy control and object recognition by compliant multi-fingered passively adaptive robotic gripper," Journal of Intelligent 8 Robotic Systems, vol. 82, no. 2, pp. 177187, 2016.

[18] Zbroja, Piotr, Szykiedans, Ksawery, and Credo, Wojciech, "Flexible grippers for industrial robots - comparison of features of low-cost 3d printed component," MATEC Web Conf., vol. 254, p. 02020, 2019.

[19] M. Manz, S. Bartsch, M. Simnofske, and F. Kirchner, "Development of a self-adaptive gripper and implementation of a gripping reflex to increase the dynamic payload capacity," in International Symposium on Robotics (ISR), pp. 1-7, 2016.

[20] K. Xue, H. Qian, and Z. Sun, "Design of an adaptive mini gripper for climbing robots," in International Conference on Advanced Robotics and Mechatronics (ICARM), pp. 618-623, 2018.

[21] Z. Erickson, H. M. Clever, G. Turk, C. K. Liu, and C. C. Kemp, "Deep haptic model predictive control for robot-assisted dressing," in IEEE International Conference on Robotics and Automation (ICRA), pp. 1-8, 2018.

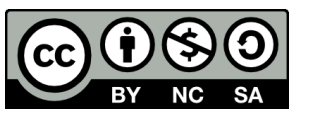
(C) 2019 by the authors. Submitted for possible open access publication under the terms and conditions of the Creative Commons Attribution CC BY-NC-SA 4.0 license (https://creativecommons.org/licenses/by-ncsa/4.0/deed.es). 